\title{
Numerical Solution of unsteady gravity flow of power-law fluids through a porous medium
}

\author{
A.W. Ogunsola, B. A. Peter \\ Department of Pure and Applied Mathematics, LAUTECH, Ogbomoso, Nigeria.
}

\begin{abstract}
In this paper, we present a numerical study of the rheological behaviour effect of non-Newtonian, power-law fluids on the unsteady gravity flow through a porous medium. The governing equations are derived and similarity solutions are determined. The finite difference method is employed to obtain solution of the nonlinear problem. The results show the existence of traveling waves. It is assumed that the viscosity is temperature dependent. We investigate the effects of velocity on the temperature field. We investigate the effect of the power-law viscosity index, and the results were discussed.
\end{abstract}

Keywords: Unsteady gravity flow; Porous media; Non - Newtonian power-law fluids and Finite difference method.

\section{Introduction}

Accurate and comprehensive computational techniques such as finite difference method can be applied to solve partial differential equations that model the flow of a porous media. Particular difficulty of the flow in porous media is that it arises, often in subtly different forms, in several separate fields of natural science and in large number of branches of technology.

Some scientists have studied gravity flow of a power-law fluids through a porous medium. These include, Peter and Ayeni[1] presented a note on unsteady temperature equation for gravity flow of a power-law fluids through a porous medium. Cortell [2] considered a paper on unsteady gravity flows of a power-law fluid through a porous medium. Olajuwon and Ayeni [3] examined a note on the flow of a power-law fluid with memory past an infinite plate. Pascal and Pascal [4] studied similarity solution to some gravity flows of non-Newtonian fluids through a porous media. Peter and Ayeni [5]investigated on the analytical solution of unsteady gravity flow of a power-law fluid through a porous medium. Zueco [6] also considered the numerical solutions for unsteady rotating high-porosity medium.

Singh[7] examined the effects of viscous dissipation and variable viscosity effects on MHD boundary layer flow in porous medium past a moving vertical plate with suction. Ogunsola and Ayeni [8] considered the temperature distribution of an Arrheniusly reacting unsteady flow through a porous medium with variable permeability. Szeri and Rajagopal [9] studied the flow of a Non-Newtonian fluid between heated parallel plates. Howarth [10] numerically considered various aspect of the Blasius flat-plate flow problem. Sparrow and Cess [11] examined the effect of magnetic field on free convection heat transfer on isothermal vertical plate. Krishnendu et al [12] studied the similarity solution of mixed convective boundary layer slip flow over a vertical plate. Hayat et al [13] they examined the effect of joule heating and thermal radiation in flow of third grade fluid over a radiative surface. In this paper, we present a numerical solution of unsteady gravity flow through a porous medium in order to see the effect of thermal conductivity expansion on the flow, proof the existence and uniqueness of the problem.

\section{Mathematical Formulation}

The governing equations are continuity, momentum equation as proposed by [2] and energy equations. Considering a two dimensional flow in the $x-z$ plane where the free surface is a streamline at a point on the surface, we expressed the flow by a modified Darcy's law.

The unsteady equations are

$$
v=-\frac{k}{\mu} \frac{\partial h}{\partial s} \quad \text { (Darcy's law) }
$$

It is a single phase flow where $\frac{\partial h}{\partial s}$ is the gradient in the flow direction and $k$ is independent of the nature of the fluid but depends on the geometry of the medium. 


$$
v=-\left(\frac{k \rho}{\mu_{e f}}\right)^{\frac{1}{n}} \frac{\partial h}{\partial s}\left|\frac{\partial h}{\partial s}\right|^{\frac{1-n}{n}} \quad \text { (Modified Darcy's law) }
$$

Where $\mathrm{S}$ is measured along the streamline,

since $z=h$ on the free surface. The rheological parameter $n$ is the power-law exponent which represents shear-thinning, i.e. $(n<1)$ and shear-thickening $(n>1)$ fluids, $k$ is the permeability, $\rho$ is the density and $\mu_{e f}$ is the effective viscosity. The Dupuit's approximation yields $\frac{\partial h}{\partial s} \cong \frac{\partial h}{\partial x}$

For small gradients which converts the problem into a one - dimensional problem. This approximation permits to assume a horizontal flow with $h=h(x, t)$ ( $\mathrm{t}$ being the time) and equation (3.12) becomes

$$
\begin{aligned}
& V_{X}=-\left(\frac{K \rho}{\mu_{e f}}\right)^{\frac{1}{n}} \frac{\partial h}{\partial x}\left|\frac{\partial h}{\partial x}\right|^{\frac{1-n}{n}} \\
& v_{r}=-\left(\frac{k \rho}{\mu_{e f}}\right)^{\frac{1}{n}} \frac{\partial h}{\partial r}\left|\frac{\partial h}{\partial r}\right|^{\frac{1-n}{n}}
\end{aligned}
$$

Where $v_{r}$ is the component of the velocity in the radial direction, whereas for radial axisymmetric flow

$$
\frac{\partial\left(h v_{r}\right)}{\partial r}=-\Phi \frac{\partial h}{\partial t}
$$

Where $\Phi$ being the porosity

The flow can also be expressed by a modified energy equation

$$
\rho c_{p} \frac{\partial T}{\partial t}=\frac{1}{r} \frac{\partial}{\partial r}\left(k(T) r \frac{\partial T}{\partial r}\right)+\mu\left(\frac{\partial v_{r}}{\partial r}\right)^{2}
$$

where $k=k(T)$

Assume that $k(T)=k_{0} e^{-\alpha T}$

$\mu=m\left(-\frac{\partial u}{\partial y}\right)^{n}, T=$ temperature, $c_{p}=$ specific heat at constant pressure, $\mu=$ viscosity, $k=$ thermal conductivity, $e^{T}$ is the thermal expansion, $\alpha$ is the coefficient of thermal expansion, $\rho=$ density, rheological parameter $n$ = power-law index and $\boldsymbol{\eta}=$ apparent viscosity.

Neglecting the dissipation term, then equation (2.6) becomes

$$
\rho c_{p} \frac{\partial T}{\partial t}=\frac{1}{r} \frac{\partial}{\partial r}\left(k_{0} e^{-\alpha T} r \frac{\partial T}{\partial r}\right)
$$

$T(r, 0)=T_{0}$

$T(0, t)=T_{1}, t>0$

$T(\infty, t)=T_{0}$

Let us introduce dimensionless variables (Non-dimensionalize)

$t^{\prime}=\frac{t}{t_{0}}, \theta(r, t)=\frac{T-T_{0}}{T_{1}-T_{0}}, r^{\prime}=\frac{r}{R}$

Substituting (2.9) into Equation (2.7) together with boundary conditions (2.8) we obtain 


$$
\begin{aligned}
\frac{\partial \theta}{\partial t^{\prime}} & =-a \alpha e^{-\alpha_{2} \theta} \frac{t_{0}}{\rho c_{p}} \frac{T_{1}-T_{0}}{R^{2}}\left(\frac{\partial \theta}{\partial r^{\prime}}\right)^{2}+a e^{-\alpha_{2} \theta} \frac{t_{0}}{R} \frac{1}{\rho c_{p}} \frac{1}{r^{\prime} R} \frac{\partial \theta}{\partial r^{\prime}}+a e^{-\alpha_{2} \theta} \frac{t_{0}}{R} \frac{1}{\rho c_{p}} \frac{\partial^{2} \theta}{\partial\left(r^{\prime}\right)^{2}} \\
& =-a_{1} e^{-\alpha_{2} \theta}\left(\frac{\partial \theta}{\partial r^{\prime}}\right)^{2}+\frac{b}{r^{\prime}} e^{-\alpha_{2} \theta} \frac{\partial \theta}{\partial r^{\prime}}+c e^{-\alpha_{2} \theta} \frac{\partial^{2} \theta}{\partial\left(r^{\prime}\right)^{2}}
\end{aligned}
$$

where $a_{1}=a \alpha \frac{t_{0}}{\rho c_{p}} \frac{T_{1}-T_{0}}{R^{2}}, b=a \frac{1}{R^{2}} \frac{t_{0}}{\rho c_{p}}, c=\frac{a}{R} \frac{t_{0}}{\rho c_{p}}$

dropping prime to get

$\frac{\partial \theta}{\partial t}=-a_{1} e^{-\alpha_{2} \theta}\left(\frac{\partial \theta}{\partial r}\right)^{2}+\frac{b}{r} e^{-\alpha_{2} \theta} \frac{\partial \theta}{\partial r}+c e^{-\alpha_{2} \theta} \frac{\partial^{2} \theta}{\partial r^{2}}$

Transforming the boundary conditions, we have

$\theta(r, 0)=0, \theta(0, t)=1, \theta(\infty, t)=0$

Introducing new variables

$\theta(r, t)=t^{\gamma} g(\eta), \eta=r t^{\beta}$

Substituting equation (2.13) into (2.11) to get

$\beta \eta^{2} g^{\prime}=-e^{-\alpha_{2} g}\left[a_{1} \eta\left(g^{\prime}\right)^{2}-b g^{\prime}-c \eta g^{\prime \prime}\right]$

$g(0)=1$

$g(\infty)=0, \eta \in(0, \infty)$

\section{Method Of Solution}

In order to solve the problem and keep it tractable, the set of non-linear ordinary differential equations (2.14) with boundary conditions in (2.15)-(2.15) have been solved analytically and numerically by using finite difference method. The computations were done by Maple 13 .

\section{Case 1: Existence and uniqueness}

We prove existence and uniqueness theorem, the problem has a solution and the solution is unique.

From equation(2.17)

when $\beta \neq 0$ equation becomes

$$
\beta \eta g^{\prime}+e^{-\alpha_{2} g}\left[a_{1} \eta\left(g^{\prime}\right)^{2}-b g^{\prime}-c \eta g^{\prime \prime}\right]=0
$$

Divide (3.1) through by $-e^{-\alpha_{2} g}$ we obtain

$$
\begin{aligned}
& \beta \eta g^{\prime} / e^{-\alpha_{2} g}+\left[a_{1} \eta\left(g^{\prime}\right)^{2}-b g^{\prime}-c \eta g^{\prime \prime}\right]=0 \\
& \frac{\beta \eta g^{\prime}}{e^{-\alpha_{2} g}}+a_{1} \eta\left(g^{\prime}\right)^{2}-b g^{\prime}=c \eta g^{\prime \prime} \\
& \frac{\beta g^{\prime}}{c e^{-\alpha_{2} g}}+\frac{a_{1} \eta\left(g^{\prime}\right)^{2}-b g^{\prime}}{c \eta}=g^{\prime \prime}
\end{aligned}
$$

\section{Theorem:}

$$
\beta \eta g^{\prime} / e^{-\alpha_{2} g}+\left[a_{1} \eta\left(g^{\prime}\right)^{2}-b g^{\prime}-c \eta g^{\prime \prime}\right]=0
$$

Which satisfies

$$
\begin{aligned}
& g(1)=1 \\
& g(\infty)=0
\end{aligned}
$$


$g^{\prime}(1)=-0.1$

Problem (3.4)-(3.7) has a unique solution

Proof:

Let

$x_{1}=\eta, x_{2}=g, x_{3}=g^{\prime}$

Then

$\frac{\beta g^{\prime}}{c e^{-\alpha_{2} g}}+\frac{a_{1} \eta\left(g^{\prime}\right)^{2}-b g^{\prime}}{c \eta}=g^{\prime \prime}$

The system of equations can be written in vector form using

$x_{1}=\eta, x_{2}=g, x_{3}=g^{\prime}$

As

$\begin{array}{ll}x_{1}^{\prime}=1 & =f_{1}\left(x_{1}, x_{2}, x_{3}\right) \\ x_{2}^{\prime}=x_{3} & =f_{2}\left(x_{1}, x_{2}, x_{3}\right)\end{array}$

$x_{2}^{\prime}=x_{3} \quad=f_{2}\left(x_{1}, x_{2}, x_{3}\right)$

$x_{3}^{1}=\frac{\beta x_{3}}{c e^{-\alpha_{2} x_{2}}}+\frac{\left(a_{1} x_{1} x_{3}-b\right) x_{3}}{c x_{1}} \quad=f_{3}\left(x_{1}, x_{2}, x_{3}\right)$

$\frac{\partial f_{1}}{\partial x_{1}}=0, \frac{\partial f_{1}}{\partial x_{2}}=0, \frac{\partial f_{1}}{\partial x_{3}}=0, \frac{\partial f_{2}}{\partial x_{1}}=0, \frac{\partial f_{2}}{\partial x_{2}}=0, \frac{\partial f_{2}}{\partial x_{3}}=1$

$\frac{\partial f_{3}}{\partial x_{1}}=\frac{-b c x_{3}}{\left(c x_{1}\right)^{2}}, \frac{\partial f_{3}}{\partial x_{2}}=\frac{\alpha_{2} x_{3} \beta}{c e^{-\alpha_{2} x_{2}}}, \frac{\partial f_{3}}{\partial x_{3}}=\frac{2 a_{1} x_{1} x_{3}-b}{c x_{1}}$

Satisfying

$1 \leq x_{1} \leq \infty$

$-k_{2} \leq x_{2} \leq k_{2}$

$-\alpha_{1} \leq x_{3} \leq \alpha_{1}$, i.e $-1 \leq \alpha_{1} \leq 1$

$g(1)=1$

$g^{\prime}(1)=-0.1$

Then

||$\frac{\partial f_{1}}{\partial x_{1}}|=0,| \frac{\partial f_{1}}{\partial x_{2}}|=0,| \frac{\partial f_{1}}{\partial x_{3}}|=0,| \frac{\partial f_{2}}{\partial x_{1}}|=0,| \frac{\partial f_{2}}{\partial x_{2}}|=0,| \frac{\partial f_{2}}{\partial x_{3}} \mid=1$

$\left|\frac{\partial f_{3}}{\partial x_{2}}\right| \leq\left|\frac{\alpha_{2} x_{3} \beta}{c e^{-\alpha_{2} x_{2}}}\right| \leq\left|\frac{\alpha_{1} \alpha_{2} \beta}{c e^{-\alpha_{2} k_{2}}}\right|$

$\left|\frac{\partial f_{3}}{\partial x_{1}}\right| \leq\left|\frac{\beta x_{3}}{c e^{-\alpha_{2} x_{2}}}-\frac{\left(b x_{3}\right)}{c x_{1}^{2}}\right| \leq\left|\frac{\alpha_{1} \beta}{c e^{-\alpha_{2} k_{1}}}+\frac{b \alpha_{1}}{c}\right|$

$\left|\frac{\partial f_{3}}{\partial x_{3}}\right| \leq\left|\frac{\beta}{c e^{-\alpha_{2} x_{2}}}+\frac{2 a_{1} \alpha_{1}}{c x_{1}}-\frac{b}{c x_{1}^{2}}\right| \leq\left|\frac{2 a_{1} \alpha_{1}+b}{c}+\frac{\beta}{c e^{-\alpha_{2} k_{2}}}\right|$

$\left|\frac{\partial f_{3}}{\partial x_{1}}\right| \leq\left|\frac{\beta x_{3}}{c e^{-\alpha_{2} x_{2}}}-\frac{\left(b x_{3}\right)}{c x_{1}^{2}}\right| \leq\left|\frac{\alpha_{1} \beta}{c e^{-\alpha_{2} k_{1}}}\right|$

$\left|\frac{\partial f_{3}}{\partial x_{3}}\right| \leq\left|\frac{\beta}{c e^{-\alpha_{2} x_{2}}}+\frac{2 a_{1} \alpha_{1}}{c x_{1}}-\frac{b}{c x_{1}^{2}}\right| \leq\left|\frac{\beta}{c e^{-\alpha_{2} k_{2}}}\right|$ 
$\left|\frac{\partial f_{3}}{\partial x_{2}}\right| \leq\left|\frac{\alpha_{2} x_{3} \beta}{c e^{-\alpha_{2} x_{2}}}\right| \leq\left|\frac{\alpha_{1} \alpha_{2} \beta}{c e^{-\alpha_{2} k_{2}}}\right|$

The upper bound of $k$, i.e.

$$
\begin{aligned}
& K(\max )=\left|\frac{\partial f_{3}}{\partial x_{2}}\right| \leq\left|\frac{\alpha_{1} \alpha_{2} \beta}{c e^{-\alpha_{2} k_{2}}}\right| \\
& \therefore K=\left|\frac{\alpha_{1} \alpha_{2} \beta}{c e^{-\alpha_{2} k_{2}}}\right| .
\end{aligned}
$$

$K<\infty$, since $c, \alpha_{2}$ and $k_{2}$ are constants other than zero. Hence $k$ exists.

Hence $\left|\frac{\partial f_{i}}{\partial x_{j}}\right|, i, j=1,2,3 \quad$ are Lipchitz continuous and are bounded in D for every bounded $x_{1}$ and $x_{2}$. Therefore; problem (3.4)-(3.7) has a unique solution. This completes the proof.

\section{Case 2}

From equation(2.17)

Let $\beta<<1$ to get

$$
g^{\prime \prime}=g^{\prime}\left(a_{1} \eta g^{\prime}-b\right) / c \eta
$$

\section{Theorem:}

Let

$$
a_{1} \eta\left(g^{\prime}\right)^{2}-b g^{\prime}-c \eta g^{\prime \prime}=0
$$

Which satisfies

$$
g(\eta)=1
$$

$g^{\prime}(\eta)=\gamma$

Where $\eta=1, \gamma$ is a guessed value

Problem (3.16)-(3.18) has a unique solution

Proof:

Let

$$
\begin{aligned}
& x_{1}=\eta \\
& x_{2}=g \\
& x_{3}=g^{\prime}
\end{aligned}
$$

Then

$$
g^{\prime \prime}=g^{\prime}\left(a_{1} \eta g^{\prime}-b\right) / c \eta
$$

The system of equations can be written in vector form using

$$
\begin{aligned}
& x_{1}=\eta \\
& x_{2}=g \\
& x_{3}=g^{\prime}
\end{aligned}
$$

As

$$
\begin{array}{lr}
x_{1}^{\prime}=1 & =f_{1}\left(x_{1}, x_{2}, x_{3}\right) \\
x_{2}^{\prime}=x_{3} & =f_{2}\left(x_{1}, x_{2}, x_{3}\right) \\
x_{3}^{\prime}=\frac{\left(a_{1} x_{1} x_{3}-b\right) x_{3}}{c x_{1}} & =f_{3}\left(x_{1}, x_{2}, x_{3}\right)
\end{array}
$$


Satisfying

$1 \leq x_{1} \leq \infty$

$-k_{2} \leq x_{2} \leq k_{2}$

$-\alpha_{1} \leq x_{3} \leq \alpha_{1}$, i.e $-1 \leq \alpha_{1} \leq 1$

$g(1)=1$

$g^{\prime}(1)=\gamma=-0.1$

Then

||$\frac{\partial f_{1}}{\partial x_{1}}|=0,| \frac{\partial f_{1}}{\partial x_{2}}|=0,| \frac{\partial f_{1}}{\partial x_{3}}|=0,| \frac{\partial f_{2}}{\partial x_{1}}|=0,| \frac{\partial f_{2}}{\partial x_{2}} \mid=0$

$\left|\frac{\partial f_{2}}{\partial x_{3}}\right|=1,\left|\frac{\partial f_{3}}{\partial x_{2}}\right|=0$

$\left|\frac{\partial f_{3}}{\partial x_{1}}\right| \leq\left|\frac{-\left(-b \alpha_{1}\right)}{c}\right| \leq\left|\frac{b \alpha_{1}}{c}\right|$

$\left|\frac{\partial f_{3}}{\partial x_{3}}\right| \leq\left|\frac{2 a_{1} \alpha_{1}-b}{c}\right| \leq\left|\frac{2 a_{1} \alpha_{1}+b}{c}\right|$

$\left|\frac{\partial f_{3}}{\partial x_{1}}\right| \leq\left|\frac{b \alpha_{1}}{c \infty}\right| \leq|0|=0$

$\left|\frac{\partial f_{3}}{\partial x_{3}}\right| \leq\left|\frac{2 a_{1} x_{3}}{c}-\frac{b}{x_{1}}\right| \leq\left|\frac{2 a_{1} \alpha_{1}}{c}\right|$

The upper bound of $\mathrm{k}$, i.e.

$K(\max )=\left|\frac{\partial f_{3}}{\partial x_{3}}\right| \leq\left|\frac{2 a_{1} \alpha_{1}}{c}\right|$

$\therefore K=\left|\frac{2 a_{1} \alpha_{1}}{c}\right|<\infty$, since $c \neq 0$. Hence $k$ exists.

Hence $\left|\frac{\partial f_{i}}{\partial x_{j}}\right|, i, j=1,2,3$ are Lipchitz continuous and are bounded in $\mathrm{D}$ for every bounded $x_{1}$ and $x_{2}$. Therefore; problem (3.16)-(3.18) has a unique solution. This completes the proof.

\section{Case 3}

From equation(3.2)

For $a_{1}>0$ we obtain

$0=b g^{\prime}+c \eta g^{\prime \prime}$

Integrating equation (3.25) we obtain

$g^{\prime}=\eta^{\frac{-b}{c}} A$

Integrating Eq.(3.26) again to get

$g=\frac{A c}{c-b} \eta^{\frac{-b+c}{c}}+B$

$\eta \in(0, \infty)$

From equation (3.27) together with the boundary conditions (3.21)-(3.22) we obtain 


$$
g(\eta)=-0.1 \frac{c}{c-b} \eta^{\frac{c-b}{c}}+1+\frac{(o .1) c}{c-b}
$$

\section{Results}

Numerical solutions of equations(3.28) together with the boundary conditions (3.21)-(3.22) were provided for various parameters in the flow equations.

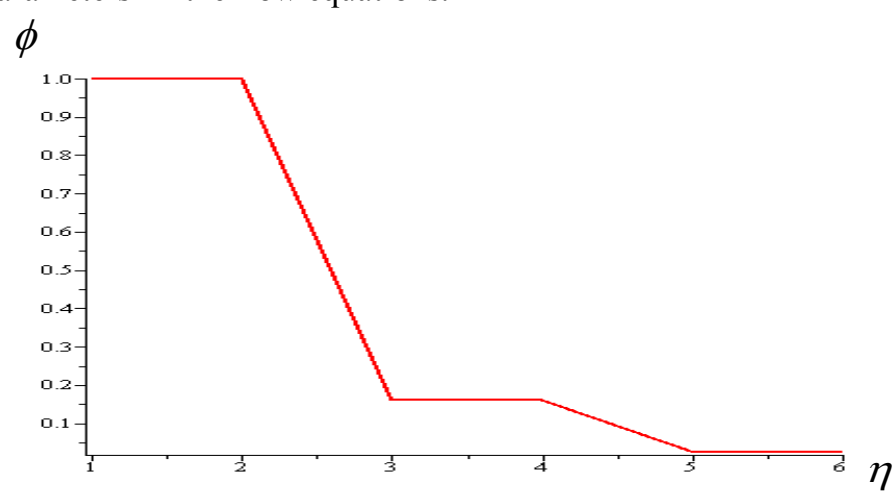

Figure 1: Graph of the temperature function $g$ against the similarity variable $\eta \quad c=0.4, b=1.6$

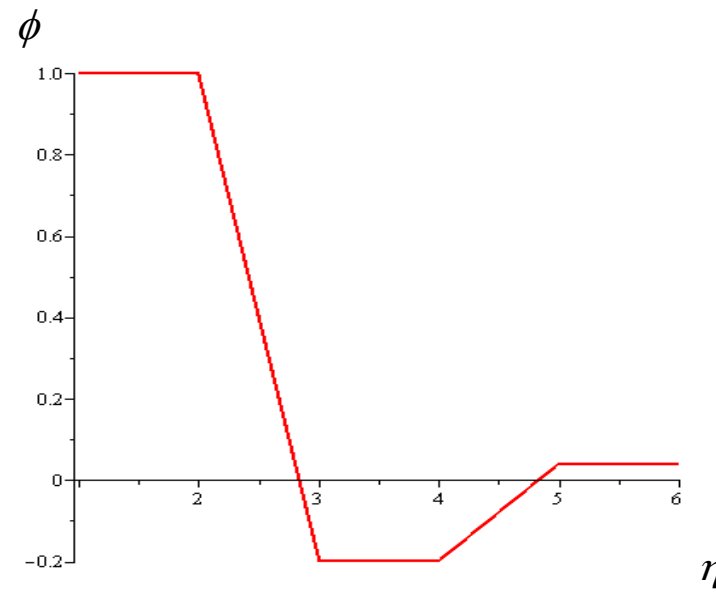

Figure 2: Graph of the temperature function $g$ against the similarity variable $\eta \quad c=-0.5, b=-2.0$

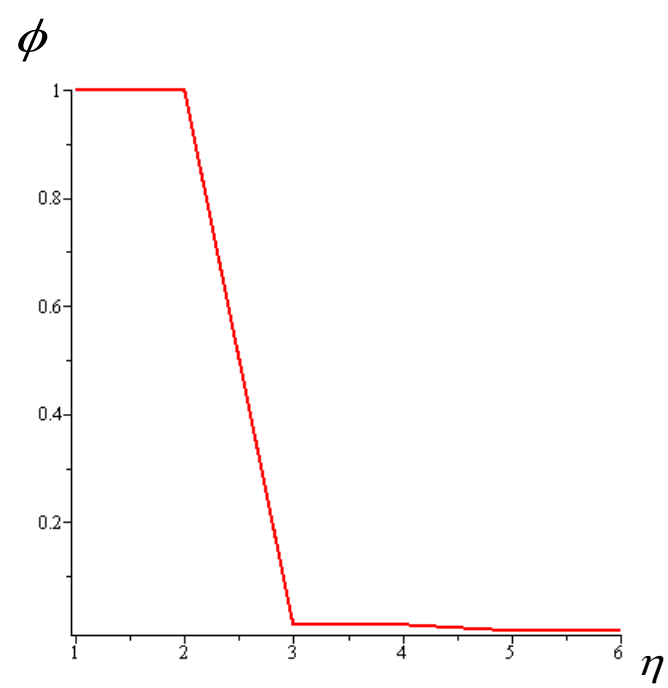

Figure 3: Graph of the temperature function $g$ against the similarity variable $\eta \quad c=0.5, b=0.1$ 


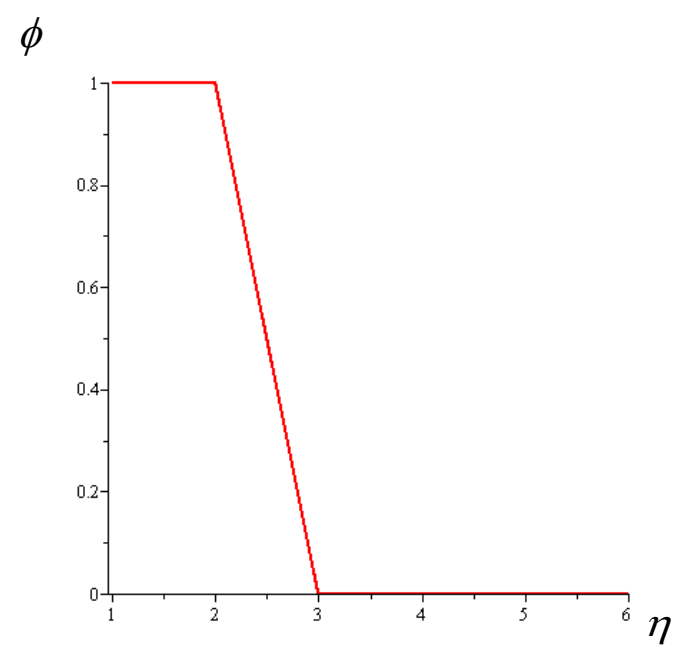

Figure 4: Graph of the temperature function $g$ against the similarity variable $\eta \quad c=0.05, b=-0.001$

\section{Conclusion}

A set of non-linear coupled differential equations governing the fluid temperature is solved analytically and numerically. A comprehensive set of graphical results for temperature is presented and discussed. We show that the problem has a solution and the solution is unique. On the other hand, we also see that the parameter $b$ affects both the flow characteristics and the accuracy of the approximate solutions significantly.

\section{References}

[1] B. A. Peter and R.O.Ayeni(2011): A note on unsteady temperature equation for gravity flow of a power-law fluids through a porous medium. Journal of the Nigerian Association of Mathematical Physics (NAMPS). Vol.18, pp 181-186.

[2] R. Cortell Bataller (2008): Unsteady gravity flows of a power-law fluid through a porous medium. Applied Mathematics and Computation. 196, pp356-362.

[3] B.I.Olajuwon and R.O.Ayeni(2004):Flow of a power-law fluid with memory past an infinite plate. Journal of the Nigerian Association of Mathematical Physics (NAMPS). Vol.8, pp 161.

[4] J.P. Pascal and H. Pascal (1993): Similarity solutions to some gravity flows of non-Newtonian fluids through a porous medium, Int. J. Non-Linear Mech. 38, pp 157 - 167.

[5] B.A.Peter and R.O.Ayeni(2011): Analytical solution of unsteady gravity flow of a power-law fluids through a porous medium. Journal of the Nigerian Association of Mathematical Physics (NAMPS), Vol.19 pp 223-228.

[6] J.Zueco (2009): Numerical solutions for unsteady rotating high porosity medium. Journal of Physical Sciences. Vol.80, Number 3.

[7] Singh (2013): Viscous dissipation and variable viscosity effects on MHD boundary layer flow in porous medium past a vertical plate with suction. Int. Journal of Engineering Science and Technology. http:/www.waset.org/journals/ijens/v4-3-30. Accessesed 11th June, 2013.

[8] Ogunsola, A.W. and Ayeni, R.O.(2007).Temperature distribution of an Arrheniusly reacting unsteady flow through a porous medium with variable permeability. Research Journal of Applied Sciences, Issue 2(4), 400-403.

[9] A.Z. Szeri and K.R. Rajagopal, Flow of a Non-Newtonian fluid between heated parallel plates. Int. J. Non-Linear Mechanics, Vol.20.No. 2. 91-101, 1985 .

[10] Howart L, on the solution of the Laminar boundary layer equations. Proc Roy Soc London A 1938;164:547-557.

[11] Sparrow EM and Cess RD, The effects of magnetic field on free convection heat transfer. Int. J. Heat Mass Transfer,3(1961),264-774.

[12] Krishnendu Bhattacharyya, Swati Mukhopadhyay and G.C.Layek, Similarity solution of mixed convective boundary layer slip flow over a vertical plate. Ain Shams Engineering Journal 4(2013),299-305.

[13] Hayat Tasawar, AnumShafig and Ahmed Alsaedi (2014) Effect of Joule heating and thermal radiation in flow of third grade fluid over radiative surface. Nonlinear Analysis and Applied Mathematics Research Group, Faculty of Science, Saudi Arabia.9(1), 1-12. http:/www.plosone.org . Accessesed March,2014. 